\title{
-Originals-
}

\section{Enlargement of bone tunnels after anterior cruciate ligament reconstruction}

\author{
Yoshihito Nakayama, Yasumasa Shirai, Tetsuya Narita and Atsusi Mori \\ Department of Orthopedic Surgery, Nippon Medical School
}

\begin{abstract}
We measured the width of tibial bone tunnels after anterior cruciate ligament (ACL) reconstruction, and investigated the correlation between bone tunnel enlargement and clinical results. Thirty-two patients who had undergone ACL reconstruction with patellar tendons augmented by woven polyester underwent physical and radiographic examinations postoperatively. The interval between surgery and examination ranged from 60 to 91 months with a mean of 68.9 months. The clinical results investigated included the total Lysholm score, the injured-to-uninjured difference in anterior knee laxity from KT-1000 arthrometer measurements, and range of motion. The width of the tibial bone tunnels was increased by a mean of $2.6 \pm 1.5 \mathrm{~mm}$ in the antero-posterior view, and by a mean of $2.7 \pm 1.4 \mathrm{~mm}$ in the lateral view. Eighteen patients had tunnel enlargements of less than $3 \mathrm{~mm}$, and 14 patients had enlargements of $3 \mathrm{~mm}$ or more. The clinical results for patients with tunnel enlargements of less than $3 \mathrm{~mm}$ were compared with those for patients with tunnel enlargements of $3 \mathrm{~mm}$ or more. However, no significant differences were found between the two groups. We conclude that bone tunnel enlargement does not appear to adversely affect clinical results over the long term. (J Nippon Med Sch 1998; $65: 377-381$ )
\end{abstract}

Key words : anterior cruciate ligament, bone tunnel, radiography, artificial material

\section{Introduction}

The functional importance of the anterior cruciate ligament (ACL) is well recognized. Various autografts, allografts and artificial materials have been used as intra-articular substitutes for ACL reconstruction, and acceptable clinical results ${ }^{1-3}$ have been reported. However, several problems ${ }^{4}$ have been identified. Recently, enlargement of the bone tunnels ${ }^{5}$ used for graft placement has been noted following reconstruction. Roverts et al. ${ }^{6}$ reported in a 2-year follow-up of patellar tendon (PT) allografts that 8 of 44 patients showed complete dissolution of the graft and a marked bone tunnel enlargement. Ikeda et al. ${ }^{7}$ also described in a followup study of GORE-TEX artificial ligaments that 5 of 73 patients had bone tunnel enlargements of more than $10 \mathrm{~mm}$. Schulte et al. ${ }^{8}$ also noted in a one-year follow-up of PT autografts and allografts that an increased width of tibial bone tunnel was found in all cases, and that the mean increase in tunnel size was $23.8 \%$ for the autografts and $24.2 \%$ for the allografts.

The purpose of this study was to measure the width of tibial bone tunnels after ACL reconstruction with PT autografts augmented by woven polyester, and to investigate the correlation between bone tunnel enlargement and clinical results.

\section{Materials and Methods}

\section{Patient Population}

Sixty-three of 92 patients who had undergone 
ACL reconstruction with PT autografts augmented by woven polyester between 1987 and 1992 were followed by means of questionnaires. Thirty-two of them fulfilled the following selection criteria and were admitted into this study: 1) the patients who had a minimum of 5 year follow-up ; 2) the patients who were directly evaluated by physical and radiographic examinations ; 3) the patients who had the same rehabilitation programs as mentioned below.

The interval between surgery and the examinations ranged from 60 to 91 months with a mean of 68.9 months. There were 14 males and 18 females with a mean age of 25.5 years (range : 15-57 years). Twenty-four patients had associated injuries: 15 had meniscal tears, and 9 had medial colateral ligament injuries.

\section{Radiographic and Clinical Evaluations}

Bone tunnel width was measured approximately $1 \mathrm{~cm}$ from the articular surface in the anteroposterior (AP) and lateral views of the knee (Fig. 1). It was always possible to measure the width of the tibial tunnel thanks to the presence of well defined margins. In contrast, we could not often measure the width of the femoral tunnel because no such well defined margins existed. Therefore, statistical analysis was based only on the tibial tunnel in this study. The calculation of the actual width of the tunnel was as follows : actual width of tunnel $=$ measured width of tunnel/radiographic magnification of $10 \%$, and the value of tunnel enlargement $=$ actual width of tunnel-a diameter of the used drill.

The subjective clinical results were evaluated by the total Lysholm knee scoring system ${ }^{9}$ (maximum
100 pts.), consisting of 8 categories such as inatability, pain, locking, swelling, support, limp, stairs and squatting. Objective clinical evaluation included anterior knee laxity measured with a KT1000 arthrometer, and range of motion (ROM). The KT-1000 arthrometer measurements were taken at $20^{\circ}$ knee flexion with a force of 90 N. Statistical analysis was performed by analyzing variance, and $\mathrm{p}=0.05$ was accepted as the minimum level of significance.

\section{Surgical Procedures and Rehabilitation Programs}

One-third of PT with bone blocks on both ends was harvested, and tubed with absorbable sutures. Woven polyester (Leeds-Keio augmentation device) was sutured tightly around the PT to make a composite graft. The graft was passed through the tibial and femoral tunnels with a diameter of $8 \mathrm{~mm}$, and fixed to the femur with 2 staples and to the tibia with a screw.

\section{Results}

\section{Radiographic Results}

A radiographic enlargement of the tibial bone tunnel was observed in all patients (Fig. 2). The width of the bone tunnel in the AP view was increased by a mean of $2.6 \pm 1.5 \mathrm{~mm}$ (range: 1 to 6 $\mathrm{mm}$ ), and that in the lateral view was increased by a mean of $2.7 \pm 1.4 \mathrm{~mm}$ (range: 1 to $6 \mathrm{~mm}$ ). Eighteen patients had an enlargement of less than 3 $\mathrm{mm}$ in the AP or lateral view, and 14 patients $3 \mathrm{~mm}$ or more.

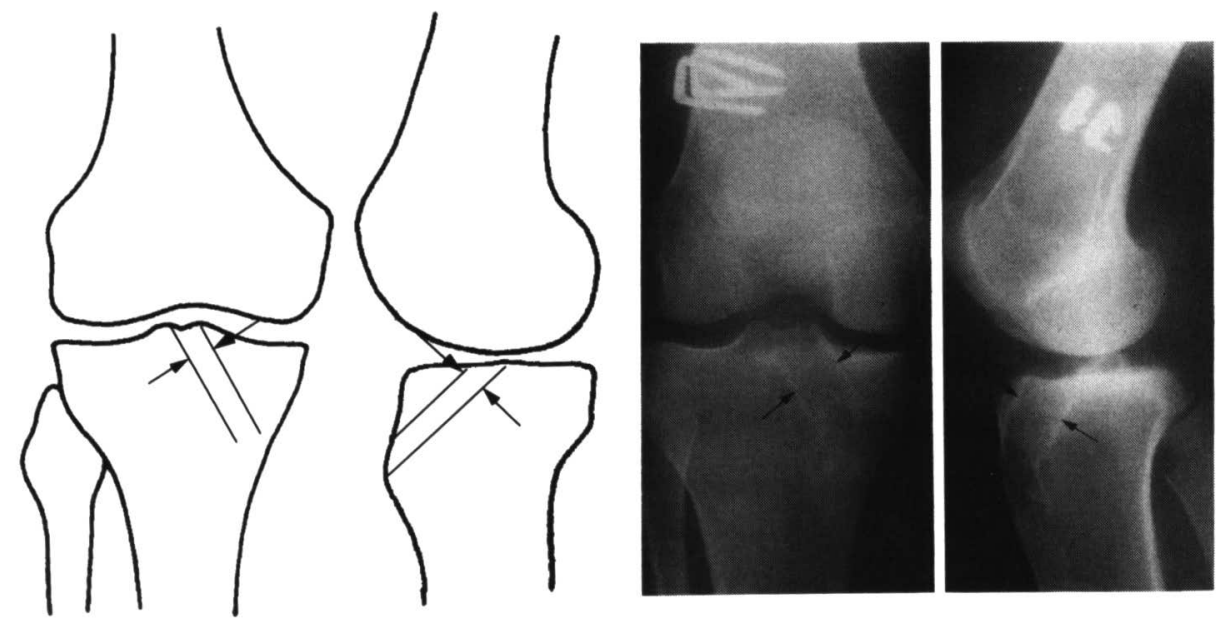

Fig. 1 Measurements of width of tibial bone tunnels 


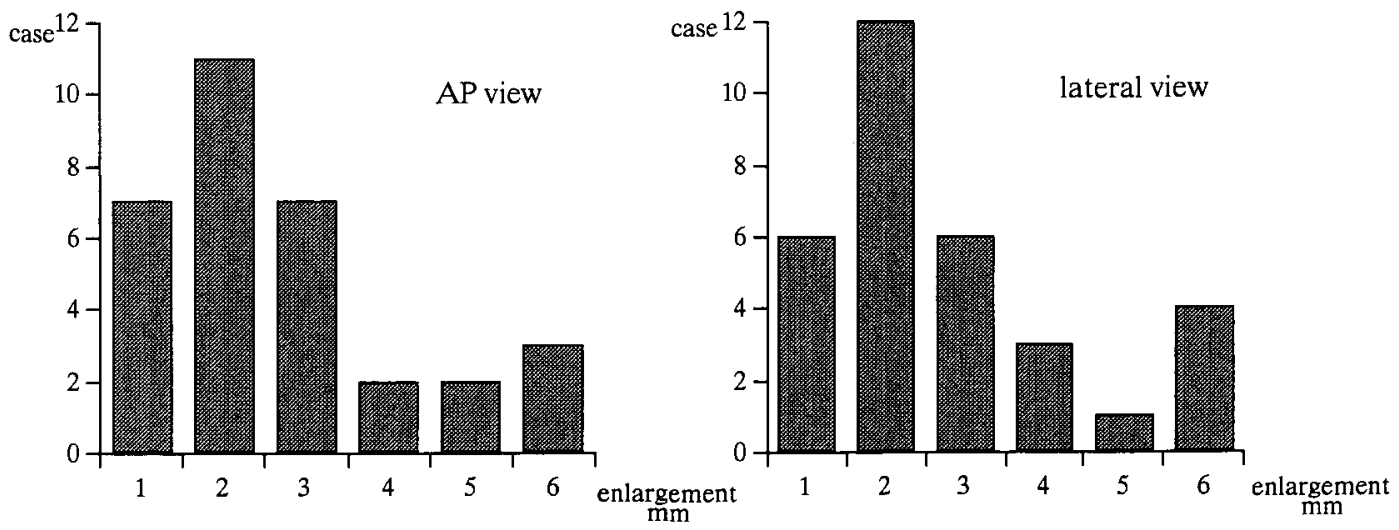

Fig. 2 Enlargement of tibial bone tunnels after ACL reconstruction

\section{Clinical Results}

The postoperative total Lysholm score ranged from 66 to $100 \mathrm{pts}$. with a mean of $95.6 \pm 7.5 \mathrm{pts}$. KT-1000 arthrometer measurements showed that the mean injured-to-uninjured difference in anterior knee laxity was $1.3 \pm 2.5 \mathrm{~mm}$ (range: -3 to 7.1 $\mathrm{mm})$. The mean extension and mean flexion were $-0.16 \pm 2.0^{\circ}$ and $149.5 \pm 2.4^{\circ}$ respectively. The mean $\mathrm{ROM}$ was $148.9 \pm 3.0^{\circ}$.

\section{Correlation between Radiological and Clinical Results}

The clinical results for patients with tunnel enlargements of less than $3 \mathrm{~mm}$ were compared with those for patients with tunnel enlargements of $3 \mathrm{~mm}$ or more. However, no signicant differences were found between the two groups (Table 1).

\section{Discussion}

Recently, bone tunnel enlargement has been reported following various surgical procedures for ACL reconstruction, although the clinical significance and etiology of bone tunnel enlargement remain obscure.

Jackson et al. ${ }^{10}$ reported in a follow-up study of PT allografts that 7 patients had chronic synovitis of the knee, and that one of them showed cystic changes in the bone tunnel. Ikeda et al. reported in a 5-year follow-up of GORE-TEX artificial ligaments that 5 of 73 patients showed a marked tunnel enlargement of $10 \mathrm{~mm}$ or more, and that the degree of enlargement was related to the patients' sporting activities and the effusion of the knee. Shimizu et al. ${ }^{11}$ stated in a report on autografts augmented by woven polyester or Kennedy LAD that the degree of tunnel enlargement was related to the postoperative anterior laxity of the knee.

In contrast, Toyoda et al. ${ }^{12}$ in a report on woven polyester reconstruction, and Linn et al. ${ }^{13}$ in a report on Achilles tendon allografts, reported that no correlation was found between tunnel enlargement and clinical results. However, they noted that long-term studies were in place to follow the patients to determine whether tunnel enlargement affects the clinical results.

In the present study, tibial tunnel enlargement was observed in all 32 patients, but no enlargement of more than $10 \mathrm{~mm}$ or cystic changes in the tunnels were observed. Moreover, the degree of enlargement was not statistically related to the clinical results in the long-term follow-up. Fahey et al. ${ }^{14}$ also stated in a study of PT autografts that modified Hughston knee score and KT-1000 arthrometer measurement of patients with an enlargement of less than $3 \mathrm{~mm}$ were compared with those with an enlargement of $3 \mathrm{~mm}$ or more, and these differences were not statistically significant. Ikeda et al. ${ }^{15}$ reported in a study of hamstring tendon autografts that no significant differences were found in the clinical results between the patients with an enlargement of less than $5 \mathrm{~mm}$ and those with that of $5 \mathrm{~mm}$ or more.

The etiology of bone tunnel enlargement is unclear, but possible explanations include either mechanical or biological causes. It is likely that drilling causes circumferential necrosis in some areas. Resorption of the necrotic bone would cause tunnel enlargement. An additional explanation could be the motion of the grafts within the tunnel. 
Table 1 Correlation between bone tunnel enlargements and clinical results

\begin{tabular}{l|ccc}
\hline \multicolumn{1}{c|}{ Clincal results } & $\begin{array}{c}\text { Enlargement }<3 \mathrm{~mm} \\
(\mathrm{n}=18)\end{array}$ & $\begin{array}{c}\text { Enlargement } \geqq 3 \mathrm{~mm} \\
(\mathrm{n}=14)\end{array}$ & Statistics \\
\hline Lysholm score & $96.4 \pm 8.3 \mathrm{pts}$. & $94.6 \pm 6.4 \mathrm{pts}$. & $\mathrm{NS}$ \\
KT-1000 measurement & $1.1 \pm 2.5 \mathrm{~mm}$ & $1.6 \pm 2.6 \mathrm{~mm}$ & $\mathrm{NS}$ \\
Range of motion & $149.2 \pm 2.6^{\circ}$ & $148.6 \pm 3.6^{\circ}$ & $\mathrm{NS}$ \\
\hline \multicolumn{3}{r}{ NS : not significant }
\end{tabular}

Friction of the grafts against the surface of the tunnel could be responsible for the tunnel enlargement.

Fahey et al. reported in a comparative study of PT autografts and allografts that the mean tibial tunnel enlargement in allografts was significantly greater than that in autografts. They noted that an immune response to the allografts was probably responsible for the occurrence of tunnel enlarge. ment. Jackson et al., in a study on ethylene oxidesterized allografts, reported that 7 patients developed persistent intra-articular reactions. Histological studies showed chronic synovitis with phagocytic cells, and removal of the allograft resulted in a resolution of the problem in all of the patients.

\section{Conclusion}

We measured the width of tibial bone tunnels after ACL reconstruction with patellar tendons augmented by woven polyester, and investigated the effects of bone tunnel enlargement on clinical results in a long-term follow-up.

1) Radiographic enlargements of the tibia were observed in all 32 patients studied. The width of the tunnel was increased by a mean of $2.6 \pm 1.5 \mathrm{~mm}$ in the AP view and by a mean of $2.7 \pm 1.4 \mathrm{~mm}$ in the lateral view. Fourteen patients had bone tunnel enlargements of $3 \mathrm{~mm}$ or more in the AP or lateral view.

2) The mean total Lysholm score, the injured-touninjured difference in anterior knee laxity from KT-1000 arthrometer measurements and ROM were $95.6 \pm 7.5 \mathrm{pts}, 1.3 \pm 2.5 \mathrm{~mm}$ and $148.9 \pm 3.0^{\circ}$ respectively .

3) There were no significant differences in clinical results between the patients with tunnel enlargements of less than $3 \mathrm{~mm}$ and those with enlargements of $3 \mathrm{~mm}$ or more.

4) It was concluded that bone tunnel enlarge- ment does not appear to adversely affect clinical results over the long term.

\section{References}

1. Fujikawa K, Matumoto H: Anterior cruciate ligament reconstruction with Leeds-Keio artificial ligament. Orthop Surg Traumatol (Japanese) 1994; 37: 657-665.

2. Nakayama Y, Shirai Y, Narita T: Long-term-follow-up study of anterior cruciate ligament reconstruction using patellar tendon augmented with woven polyester. J J Rheum Joint Surg 1996; 5-14.

3. Nakayama Y, Shirai Y, Narita T, Mori A: A followup arthroscopy after anterior cruciate ligament reconstruction using patellar tendon augmented by woven polyester. J Nippon Med Sch 1997; 67: 512-517.

4. Olson EJ, Kang JM, Georgescu HI, Mason GG, Evans $\mathrm{CH}$ : The biochimical and histological effects of artificial ligament wear particles : In vivo and in vitro studies. Am J Sports Med 1988; 16: 558-570.

5. Uemaki $H$, Hukubayashi $T$, Ikeda $K$, Shimojo $H$, Okuwaki T, Matsuda N, Hioki S, Muramatsu T: Bone tunnel enlargement of knee anterior cruciate ligament reconstruction with iliotibial tract: Reinforced Leeds-Keio or LAD artificial ligaments. Journal of Tokyo Knee Society (Japanese) 1994; 15: $83-86$.

6. Roberts TS, Drez D, Mccarthy W, Paine R: Anterior cruciate ligament reconstruction using freeze-dried, ethylene oxide-sterilized,bone-patellar tendon-bone allografts. Am J Sports Med 1991; 19:35-41.

7. Ikeda K, Hukubayashi $T$, Okinaga S, Koide S: Complication of the GORE-TEX artificial ligament: Special emphasis on the bony tunnel enlargement. Journal of Tokyo Knee Society (Japanese) 1993; 14 : 103-106.

8. Schulte K, Majewski M, Irrgang JJ, Fu FH, Harner $\mathrm{CD}$ : Radiographic tunnel changes following arthroscopic ACL reconstruction: Autograft versus allograft. Arthroscopy 1995; 11: 372-373.

9. Tegnar Y, Lysholm J, Gillquest J: Rating systems in the evaluation of knee ligament injuries. Clin Orthop 198: 43-49, 1985.

10. Jackson DW, Windler GE, Simon TM: Intraarticular reaction associated with the use of freezedried, ethylene oxide-sterilized, bone-patellar 
tendon-bone allografts in the reconstruction of the anterior cruciate ligament. Am J Sports Med 1990; 18: $1-11$.

11. Shimizu H, Oomori K, Okamoto K, Yamaji Y, Hamawaki J: Analysis of roentgenogram after $\mathrm{ACL}$ reconstruction. J Chubu Jpn Assoc Orthop Traumatol (Japanese) 1992; 35: 165-166.

12. Toyoda K, Matsumoto H, Otani T, Tokunaga Y, Sasazaki Y, Inoue M, Ushihisa N, Sekiguchi O, Nakayama S, Hujikawa K: Changes in bone tunnel diameters after anterior cruciate ligament reconstruction with Leeds-Keio artificial ligament. Journal of Tokyo Knee Society (Japanese) 1977; 18: $92-95$.
13. Linn RM, Fischer DA, Smith JP, Burstein DB, Quick $\mathrm{DC}$ : Achilles tendon allograft reconstruction of the anterior cruciate ligament-deficient knee. Am J Sports Med 1993; 21: 825-831.

14. Fahey M, Indelicate PA: Bone tunnel enlargement after anterior cruciate ligament replacement. Am J Sports Med 1994; 22: 410-414.

15. Ikeda $\mathrm{H}$, Niga S, Hoshino A, Asahina S, Tei $\mathrm{K}$, Nagatsuka Y: Bone tunnel enlargement after anterior cruciate ligament reconstruction. Journal of Tokyo Knee Society (Japanese) 1977; 18: 88-91.

(Received, June 11, 1998)

(Accepted for publication, July 15, 1998) 